\title{
Erratum à: Le vidéolaryngoscope permet une intubation avec un tube à double lumière plus rapide que le laryngoscope standard
}

\section{Erratum to: Faster double-lumen tube intubation with the videolaryngoscope than with a standard laryngoscope}

\author{
Mustapha Bensghir, MD • Hassan Alaoui, MD • Hicham Azendour, PhD • \\ Mohamed Drissi, MD • Abderhmane Elwali, MD • Mohamed Meziane, MD • \\ Jaafar Salim Lalaoui, PhD • Ali Akhaddar, PhD • Nouredine Drissi Kamili, PhD \\ Published online: 2 February 2011 \\ (C) Canadian Anesthesiologists' Society 2011
}

Erratum to: Can J Anesth/J Can Anesth DOI 10.1007/s12630-010-9372-7

Une erreur s'est glissée dans l'article intitulé «Le vidéolaryngoscope permet une intubation avec un tube à double lumière plus rapide que le laryngoscope standard» publié dans le numéro du mois de novembre 2010, Can J Anesth 2010; 57: 980-984. Au tableau 2, page 983, dans la colonne du Groupe VL, le temps d'intubation $(\mathrm{sec})$ devrait se lire $39,9 \pm 4,4$ et dans la colonne du Groupe LS, le temps d'intubation (sec) devrait se lire $47,9 \pm 5,4$.

La version en ligne de l'article original est disponible à l'adresse suivante / sous doi:10.1007/s12630-010-9372-7.

M. Bensghir, MD $(\varangle) \cdot$ H. Alaoui, MD $\cdot$ M. Drissi, MD

A. Elwali, MD · M. Meziane, MD · J. S. Lalaoui, PhD .

N. D. Kamili, PhD

Du Service d'Anesthésiologie, Hôpital Militaire Med V Rabat,

Boite postale 8840, Rabat Agdal Rabat, Maroc

e-mail: mustaphabens_15rea@hotmail.com 